\title{
Intra/Inter-Particle Energy Transfer of Luminescence Nanocrystals for Biomedical Applications
}

\author{
Ching-Ping Liu, ${ }^{1}$ Shih-Hsun Cheng, ${ }^{1}$ Nai-Tzu Chen, ${ }^{1,2}$ and Leu-Wei Lo ${ }^{1}$ \\ ${ }^{1}$ Division of Medical Engineering Research, National Health Research Institutes, Zhunan 350, Taiwan \\ ${ }^{2}$ Department of Chemistry, National Taiwan University, Taipei 106, Taiwan
}

Correspondence should be addressed to Leu-Wei Lo, lwlo@nhri.org.tw

Received 17 February 2012; Accepted 11 April 2012

Academic Editor: Patricia Murray

Copyright (C) 2012 Ching-Ping Liu et al. This is an open access article distributed under the Creative Commons Attribution License, which permits unrestricted use, distribution, and reproduction in any medium, provided the original work is properly cited.

\begin{abstract}
Elaborate design of energy transfer systems in luminescent nanocrystals revealed tremendous advantages in nanotechnology, especially in biosensing and drug delivery systems. Recently, upconversion nanoparticles have been discussed as promising probes as labels in biological assays and imaging. This article reviews the works performed in the recent years using quantum dot- and rare-earth doped nanoparticle-based energy transfer systems for biomedical applications.
\end{abstract}

\section{Introduction}

Luminescence nanocrystals offer great promise in numerous biomedical applications from early diagnosis to cancer therapy. Quantum dots (QDs) have unique photophysical properties offering significant advantages as optical labels for biosensing. Unlike molecular fluorophores capable of the narrow excitation range, QDs have broad absorbance bands, which allow the excitation of various sizes QDs at a common wavelength. Owing to their bright and stable fluorescence, QDs improve sensitivity in their use as optical labels. However, the major concerns about potential toxicity of QDs have limited their use in biological applications. Therefore, searching different types of nanoparticles with better biocompatibility is emerging with considerable interest. Recently, rare-earth-doped nanoparticles were demonstrated with an appearing potential in biological applications because of their optical properties and low cytotoxicity [1-3]. Rareearth doped nanoparticles such as upconversion nanoparticles (UCNPs) have attractive features as the following: high photostability, high light penetration depth in tissue, easy separation of the narrow emission bands from stray light, good biocompatibility, and weak autofluorescence background generated by near-infrared (NIR) excitation [4]. The synthesis and investigation of UCNPs were reviewed recently by Haase and Schäfer [5], and their biological applications were reviewed by Wang et al. [4] and Zhou et al. [6] .
In this paper, we present the current use of QD- and rareearth doped nanoparticle-based energy transfer systems for biological applications. Extensive efforts are also dedicated to the research involving certain luminescence nanocrystals possessing unique properties to exploit mechanisms of intra/inter particle energy transfer in nanometer scale. To be different from those reviews focus on synthesis and development of luminescence nanocrystals, this current review put emphasis on their biological applications that deploy elaborate design of energy transfer systems such as Förster/fluorescence resonance energy transfer (FRET), bioluminescence resonance energy transfer (BRET), and luminescence resonance energy transfer (LRET) as well as photoinduced cleavage for drug release and photoactivation of drugs. The QDs for biosensing and the rare-earth-doped nanoparticles for cancer therapy are hereof reviewed in detail in attempt to reveal the advances of NIR and X-ray sensitized nanoparticles for potential deep cancer diagnostics and therapy.

\section{Luminescence-Nanocrystal-Based Energy Transfer Systems}

2.1. Quantum Dots. Quantum dot (QD) serves as a great candidate for nanodelivery and nanosensor in biological application. QDs have many advantages such as highly 
photostability and brightness as well as resistance of photobleaching as compared to conventional organic fluorophores [7-10]. Therefore, the large extinction coefficients and wide absorption wavelength ranges of QDs allow excitation of different QDs by a single excitation source. These nanosizes of QDs result in size-tunable absorption and emission wavelengths by their quantum mechanical behaviors $[7,8]$. Owing to these advantages, QDs suggest they are ideally suitable for long-term biosensors for multiple biomolecules tracking. For the photoluminescence properties of broad absorption and narrow emission (few spectral cross-talk), QDs have been wildy exploited as efficient Forster resonance energy transfer (FRET) donors to make the selection of acceptor easy. There were many scientists have designed specific peptide and protein-based conjugations of QDs and utilized FRET to sense biological behaviors and mechanisms in vitro and in vivo. In another approach, a number of reports used QD as the drug carrier and monitored the dynamics of drug release from QD using FRET [8, 11-13].

2.1.1. Biosensing. In previous reports, QDs have been used in biosensing with diverse energy transfer designs of singlestep FERT system (see Figure 1) and multiple-step FRET system (see the Figure 2). For DNA detection, Leong's group developed a single-step quantum-dot-mediated system (QDFRET) to investigate the structural composition and dynamic behavior of plasmid DNA hybrid nanostructure in vitro [14]. The QD-FRET system was developed with $605 \mathrm{~nm}$ QD as the donor and the plasmid DNA (pDNA), whereas Cy5 was conjugated as the acceptor for energy transfer. The ultrasensitive system reveals the dynamic release of DNA vectors from the hybrid nanostructure. The FRET signal was abrogated as pDNA releases into the cytosol. The protection of pDNA from potential enzymatic barriers such as nuclease-mediated DNA degradation depended on the stability of nanocarriers. By using QD-FRET system, both pDNA release and subsequent degradation could be simultaneously monitored (as shown in Figure 1(a)).

Choi et al. demonstrated a QD-peptide probe which was prepared simply from one step electrostatic interaction for detecting HIV-1 protease (HIV-1 PR) activity in vitro and in live cells (see Figure 1(b)) [15]. The peptide with HIV-1 PR recognition site SQNYPIVQ was conjugated to the negative charged QDs. It was specifically cleavable when expose to HIV-PR and such a peptide-QD system could render the detection sensitivity of HIV-PR in the range of picomol. Lee et al. also designed a positively charged compact QDDNA complex for detection of nucleic acids [16]. The fluorescence of the QD is quenched up to $90 \%$ by complexion with $5^{\prime}$-carboxytetramethylrhodamine- (TAMRA-) modified oligonucleotide through FRET. The present QDDNA probe shows the capability to detect the $200 \mathrm{~nm}$ H5N1 influenza virus oligonucleotide. QD-based molecule also designed to unveil the detailed information of DNA microenvironment via FRET. Shaheen et al. developed a de novo method for quantifying the decondensation of pDNA in heterochromatin and euchromatin separately by means of a QD-FRET technique [17]. In this system, FRET between
QD-labeled pDNA as a donor and rhodamine-labeled polycation as an acceptor was measured for visualizing and quantifying nuclear decondensation in specified nuclear subdomains via the use of an artificial DNA condenser. Chen's Group developed a new QD-aptamer (QD-apt) beacon that acts by folding-induced dissociation of a DNA intercalating dye, $\mathrm{BOBO}-3$, is demonstrated with label-free thrombin detection [18]. The beacon is constructed by (1) coupling of a single-stranded thrombin aptamer to Qdot 565 via EDC/Sulfo-NHS chemistry and (2) staining the duplex regions of the aptamer on QD with excess BOBO-3 before thrombin binding. When mixing a thrombin sample with the QD-apt beacon, BOBO-3 is competed away from the beacon due to target-induced aptamer folding, which then caused a decrease in QD FRET-mediated BOBO-3 emission and achieved thrombin quantitation.

With the mechanism as shown in Figure 2(a), Rogach's Group fabricated the hybrid nanostructure of CdTe-conjugated polymers, which can use the light harvesting and FRET donor properties of QD for DNA hybridization detection [19]. The conjugated polymer: Poly[9,9-bis (3'-((N,N-dimethyl)-N-ethylammonium)-propyl)-2,7-fluorene-alt-1,4-phenylene] dibromide (PDFD) plays two key roles in the detection function. First, PDFD is a light harvesting antenna to enhance the emission of QD by the first level FRET (FRET 1). The other is that PDFD provides a positive-charged surface to allow negative-charged dye-labeled DNA to interact with. This second FRET (FRET 2) from QD to IRD700 (infrared fluorescence-emitting dye) labeled on the DNA probe provides a sensing platform discriminating between complementary and noncomplementary DNA. Owing to the higher local charge density of ds-DNA, the unambiguous detection is allowed by a stronger electrostatic binding to the PDFD/QD complex. Therefore, the DNA hybridization could be quantified by the ratio of emission intensity of IRD700 dye to that of QD.

Recently, Chen et al. demonstrated a two-step FRET system (see Figure 2(b)) which was constructed from the QD donor to the first acceptor of a nuclear dye (ND, First energy transfer, E12), and ND served as a relay donor to the second acceptor Cy5 (second energy transfer, E23) [20, 21]. For stable compact nanocomplexes, the QD donor drives energy transfers through the ND which acted as a relay to Cy5 conjugated on the polymeric gene carrier (E12 and E23 were "on"). While the nanocomplex begins to unpack and release intact pDNA, the E23 was off (Emission of Cy5 was diminished) and only the ND was "on." Finally, the E12 was "off" due to the degradation of free pDNA. By monitoring the combinations of FRET-mediated emission from the ND and Cy5 with this two-step QD-FRET system, both polyplex dissociation and pDNA degradation within cells were sensed simultaneously.

Medintz's group has previously reported the use of metal-affinity coordination between $\mathrm{His}_{n}$ residues and the $\mathrm{ZnS}$ shell of $\mathrm{CdSe} / \mathrm{ZnS} \mathrm{QDs}$. The $\mathrm{His}_{n}$ residues have strong binding affinities for conjugating proteins, peptides, and even modified DNA sequences to the nanocrystals to sense nutrients, explosives, DNA, and enzymatic activity via FRET [22, 23]. Authors used this concept to design 


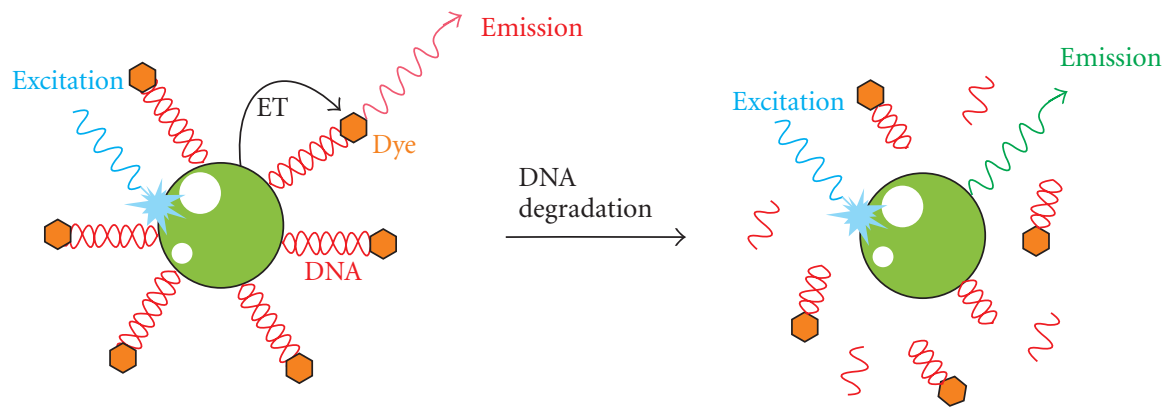

(a)

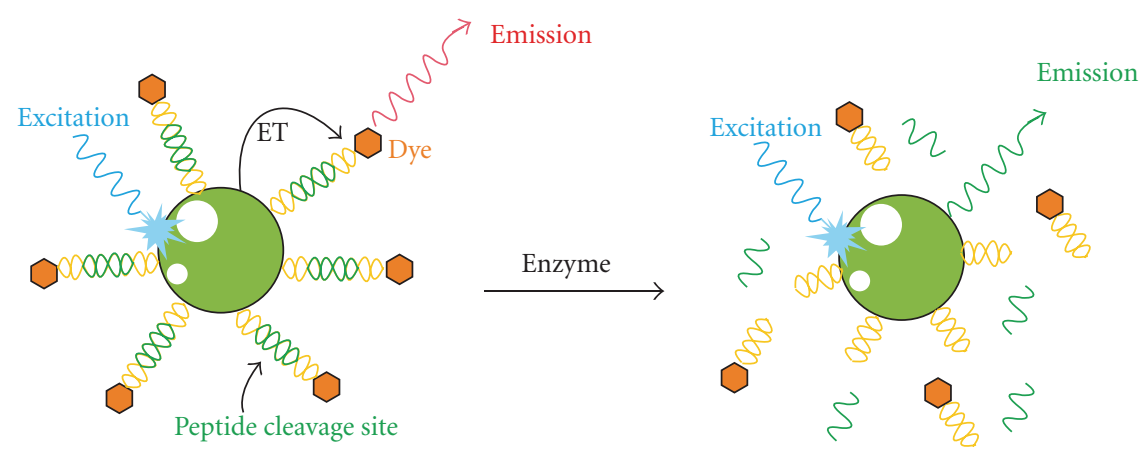

(b)

FIGURE 1: Single-step FRET-based QD biosensors designed to probe DNA degradation and protein. (a) The dsDNA (labeled with fluorescent dyes) degraded from QD surface compromises the FRET between dsDNA and QD. (b) The fluorescently labeled peptides, each with an enzyme-specific cleavage site, are bound to a QD and construct FRET. The FRET becomes decreasing as the enzyme starts to cleave the peptide into fragments.

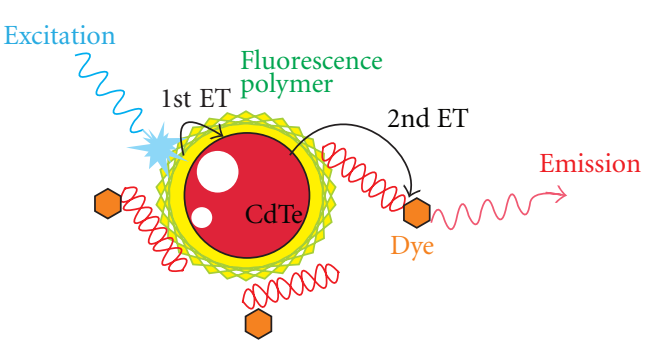

(a)

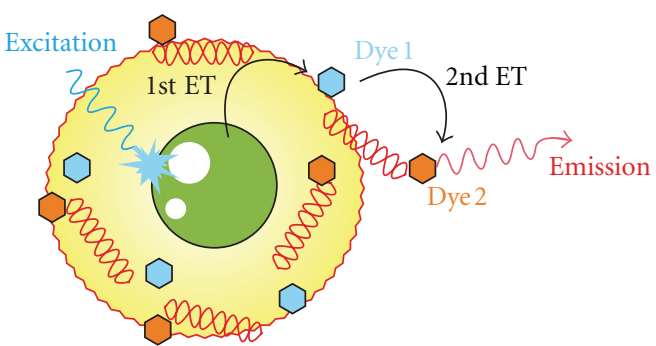

(b)

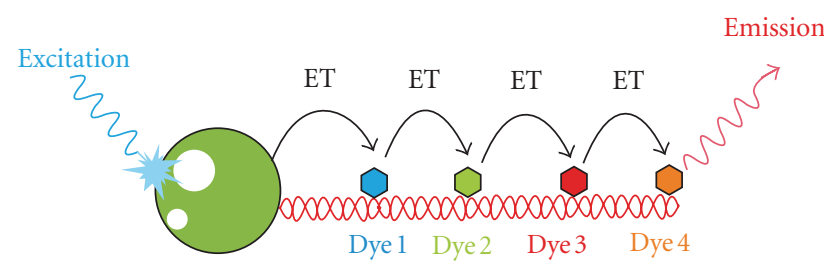

(c)

FIGURE 2: Multiple-step FRET-based QD biosensors. (a) The hybrid nanostructure of CdTe conjugated fluorescence polymers, which exploits the light harvesting and FRET donor properties of QD for detection of DNA hybridization. (b) The two-step QD-FRET system, where the first energy transfer occurs from QD to Dye 1 in polymeric matrix, followed by the second energy transfer from Dye 1 to DNA-labeling Dye 2. Thus both the polymer and DNA degradation in cells can be assessed by the combination of FRET-mediated emission. (c) The multiple dye-labeled DNA symmetrically arrays on the surface of QD. By excitation of the central QD donor, the energy transfer is relayed through the sequentially aligned dye-acceptors which emit from the visible to NIR portion of the spectrum. 
QD-fluorescence protein assemblies for sensing caspase-3 activity [24]. The QD-fluorescent protein sensor is developed as the fluorescence protein: mCherry expressing the caspase3 cleavage site self-assembled to the surface of CdSe-ZnS Dihydrolipoic acid- (DHLA-) functionalized QDs, resulting in FRET quenching of the QD and sensitized emission from the mCherry acceptor. While caspase- 3 cleaves the linker, it reduces the FRET efficiency.

Based on the [25], Medintz et al. demonstrated the $\mathrm{Ni}^{2+}$-supplemented $565 \mathrm{~nm}$ ITK-COOH QDs resulting in $\mathrm{His}_{6}$-driven protein coordination to the $\mathrm{Ni}^{2+}{ }_{-} \mathrm{COOH}$ QD surfaces in mCherry-His ${ }_{6}$ expressing cells. They used FRETsensitized emission from QD to mCherry to confirm the intracellular protein assembly. Recently, the same group demonstrated the hybrid multifluorophore DNA-photonic wires that both self-assemble around QDs and QDs function as UV energy harvesting to drive FRET cascades through the DNA wires with emission which approach near-infrared (see Figure 2(c)) [26]. The peptide portion facilitated metalaffinity coordination of multiple hybridized DNA-dye structures to a central QD completing the final nanocrystal-DNA photonic wire structure. They conjugated multiple copies of DNA hybridized with 4-sequentially arranged acceptor dyes on the central QD and demonstrated 4-consecutive energy transfer steps monitored with both steady-state and time-resolved spectroscopy. Additionally, Medintz's group investigated the use of QD-peptide FRET sensor to monitor the activity of the Botulinum neurotoxins (BoNT) serotype A light chain protease (LcA) [27]. They designed modular LcA peptide substrate with $\mathrm{Cy} 3$ acceptor and the terminal oligohistidine that allowed for peptide-QD-selfassembly. The 350 pM LcA limit of detection was demonstrated with indirect preexposure of peptide to LcA prior to QD assembly. Rao's group demonstrated that carboxylated polymer modified QDs could coordinate $\mathrm{His}_{6}$-tagged luciferase enzymes to create protease sensors via bioluminescence resonance energy transfer (BRET) [28]. While excess $\mathrm{Ni}^{2+}$ was added to the QDs, $\mathrm{His}_{6}$-luciferase binding and BRET interactions were significantly increased.

Freeman et al. reported Nile-Blue- (NB-) functionalized QDs revealed the luminescence quenching of the QDs via FRET mechanism [29]. The FRET process triggering by QDs is prohibited by colorless $\mathrm{NBH} 2$ units yielded by the reduction of the NB-units by 1,4-dihydronic-otineamide adenine dinucleotide (phosphate), NAD $(\mathrm{P}) \mathrm{H}$. This process could be used to detect the $\mathrm{NAD}(\mathrm{P}) \mathrm{H}$ cofactors, and a series of $\mathrm{NAD}(\mathrm{P})+$-dependent enzymes and their substrates. Moreover, authors used the modified QDs to follow intracellular metabolism for anticancer drugs screening, for example, the anticancer drug: Taxol which is known to inhibit the metabolic processes. They demonstrated the QD fluorescence increased by the $\mathrm{NAD}(\mathrm{P}) \mathrm{H}$ cofactors in the Taxol-treated cells.

Skajaa et al. developed a lipoprotein-based nanoparticle that consists of a quantum dot (QD) core and Cy5.5 labeled lipidic coating to study of lipoprotein-lipoprotein interaction [30]. They judiciously tuned QD/Cy5.5 ratio to optimize the FRET between QD and Cy5.5 coating. The dynamics of lipid exchange influenced by apolipoproteins is monitored via
FRET process. Moreover, authors have studied high density lipoprotein- (HDL-) cell interactions and exploit FRET to visualize HDL association with live macrophage cells.

2.1.2. Drug Delivery and Therapy. In another approach, QDs were introduced as a traceable marker and a nanocarrier for drug delivery. Bagalkot et al. reported the multifunctional QD-Apt delivery system for simultaneous imaging and therapeutic uses [31]. The multifunctional QD-Apt was conjugated with the targeting modality of RNA aptamer. The RNA aptamers serve for both targeting and therapy functions. For targeting, RNA aptamers pinpoint the prostate-specific membrane antigen (PSMA) expressed in LNCaP cells; and for therapy, anticancer drug, doxorubicin (Dox), was intercalated on aptamers. In this bi-FRET (dual donor-quencher) system, the QD fluorescence was quenched by Dox and the fluorescence of Dox was quenched by the RNA aptamers. When the Dox was loading, both QD and Dox fluorescence were turned "OFF" through bi-FRET. While the QD-Apt complex was uptaken by prostate cancer cells and Dox was gradually released, both QD and Dox fluorescence switched to "ON." This multifunctional QD-Apt-Dox delivered Dox to PSMA-expressing LNCaP cells more efficiently compared to nontargeted PC3 cells, and the delivery efficiency of Dox was monitored by FRET spontaneously.

2.2. Rare-Earth-Doped Nanoparticles. Rare-earth-doped nanoparticles usually include a host/matrix as light absorber and the rare earth ions are doped into a host of high intrinsic transparency and low vibrational energy. The rare-earthdoped nanoparticles can be either excited on the domain of host/matrix by ultraviolet (UV) light followed by the subsequent energy transfer to the rare-earth ions or using direct excitation of the rare-earth ion responsible for the emission. In the case of codoping such as $\mathrm{Ce}$ and $\mathrm{Tb}$, it is able to transfer energy from $\mathrm{Ce}^{3+}$ to the emitting ion of $\mathrm{Tb}^{3+}$. In some cases, such as UCNPs, they display the unique property of converting low-energy NIR light to highenergy UV or visible light. This process is based on sequential multiphoton absorption and energy transfer steps involving real metastable, long-lived states [32]. The intraparticle energy transfer between two doping lanthanide ions in the excited states leads to short wavelength emission.

2.2.1. Bioimaging. UCNPs conjugated with biological molecules are investigated as fluorescent markers for biological imaging due to their attractive optical properties as mentioned in Section 1. Following significant progress in techniques, UCNPs have been introduced for cell and animal imaging [33-39]. Recently, multimodal imaging and imaging combined therapies have become a new trend [40-42]. $\mathrm{NaGdF}_{4}$ codoped $\mathrm{Tm}^{3+} / \mathrm{Er}^{3+} / \mathrm{Yb}^{3+}$ upconversion nanophosphors were used to demonstrate the dual-modality of NIRto-NIR upconversion luminescence (UCL) together with MRI for in vivo imaging of the whole-body animal [43]. The $\mathrm{Gd}^{3+}$ introduced in the synthesis of UCNPs enhanced the contract of MRI, whereas the NIR-to-NIR UCL enables imaging of the target inside the tissues due to both excitation 


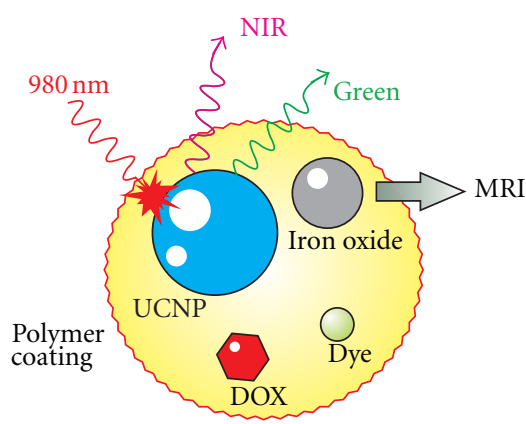

(a)

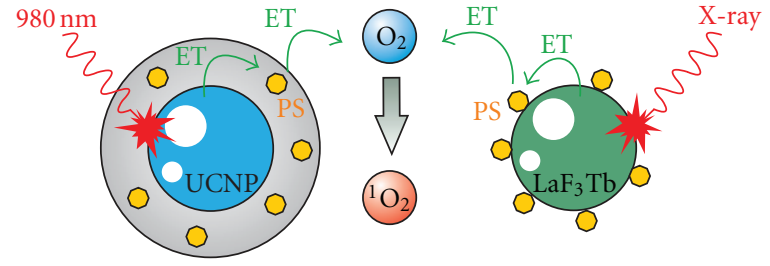

(c)

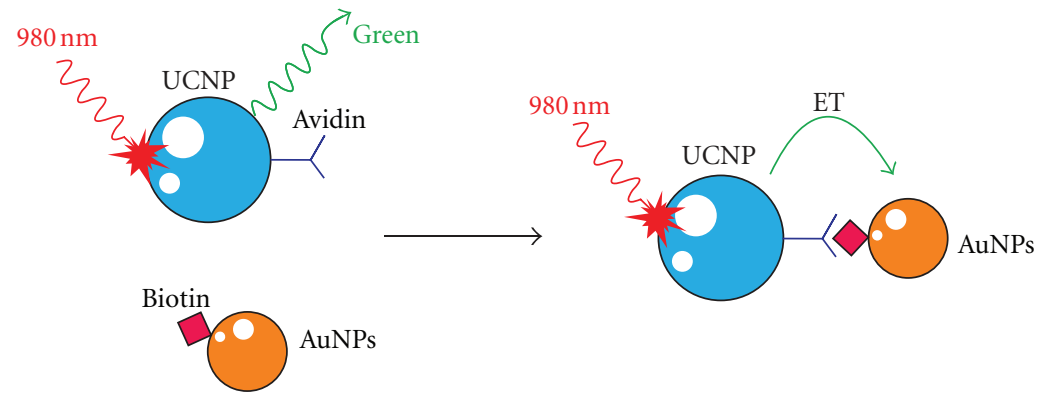

(b)

FIgURE 3: Different UCNP nanocomposites and applications. (a) Triple-modal UCL/FL/MRI nanocomposite: UCNPs for UCL, dye for FL, and iron oxide for MRI; DOX for therapeutic drug. (b) The biotin-avidin affinity system modulates FRET via the quench of UCNPs emission by gold nanoparticles. (c) Photodynamic therapy in deep tissue: both NIR excitation of UCNPs and X-ray irradiation of scintillation nanoparticles can generate visible light, which further synergistically activates photosensitizers in proximity to generate cytotoxic singlet oxygen for cancer treatment.

and emission light can penetrate deep into/from tissues. Their results showed high contrast in both NIR-to-NIR and NIR-to-visible luminescence imaging as well as MRI, which can serve as a platform technology for the next generation of probes for in vivo bioimaging.

In Figure 3(a), polymer encapsulated UCNPs and iron oxide as a nanocopmposite can be further loaded fluorescent dye and anticancer drug for triple-modal UCL/downconversion fluorescence(FL)/MRI in vitro and in vivo as well as the imaging-guided and magnetic-targeted drug delivery [44]. The Squaraine (SQ) fluorescent dye-loaded nanocomposite was used for the whole-body animal imaging of nude mice. Both strong UCL signals from UCNPs and FL from the SQ dye were observed predominately in the liver as well as a dramatic darkening effect from MRI, indicating the high uptake of the nanocomposite in the liver. On the other hand, for doxorubicin (DOX) loaded into the nanocomposite, the saturated maximal DOX loading efficiency was obtained ca. $8 \%(\mathrm{w} / \mathrm{w})$ and nearly $45 \%$ of DOX was released in the acidic condition (at $\mathrm{pH}$ 5.0). The multimodal UCL/FL/MR imaging can enhance sensitivity and improve tissue penetration compared to each single imaging tool. In addition, the imagingguided magnetic targeted drug delivery is specific and selective to localized regions, which can be extended to the delivery of NIR-light induced UCNP-based photothermal therapy as reported by the same group [45].
2.2.2. FRET-Based Biosensing. Organic dyes and QDs have been already known for some drawbacks as applied for in vitro or in vivo FRET assays due to the strong autofluorescence from cell and biomolecules. Depending on different dopants of lanthanide ions, rare-earth-doped nanoparticles typically function as energy donors, can provide tunable emission to fit any accepters in energy transfer nanocomposites. For DNA-sensitive study, Wang et al. have developed a novel DNA fluorescence detection method based on the polymer-coated rare-earth-doped nanoparticles [46]. The fluorescence originated from $\mathrm{Tb}^{3+}$ transitions of $\mathrm{LaF}_{3}: \mathrm{Ce}^{3+}$, $\mathrm{Tb}^{3+}$ nanoparticles can be quenched by the presence of nucleic acids such as DNA. They proposed two possible fluorescent quenching mechanisms: (1) the formation of hydrogen bonds between DNA and carboxylic acids outside of nanocomposites enables energy transfer from the excited $\mathrm{Tb}^{3+}$ to DNA; (2) the generation of a nonfluorescent or weakly fluorescent compound which is a fluorophorequenching ternary complex involves the $\mathrm{LaF}_{3}$ and DNA. Due to that, the concentration of DNA can be determined by measuring the fluorescence intensity of $\mathrm{LaF}_{3}: \mathrm{Ce}^{3+}, \mathrm{Tb}^{3+}$ nanoparticles.

UCNPs also have an appearing potential as donors in FRET-based application [47-51]. The UCNPs as donors can greatly improve the sensitivity and efficiency of FRET assays because most biological materials do not absorb 
NIR light thus to reduce the background interference. In addition, the extremely narrow and sharp emission bands of lanthanide ions are also helpful for the separation of cell background fluorescence and direct acceptor excitation in FRET experiments. Li's Group developed an upconversion biosensor based on the FRET between bioconjugated UCNPs and gold NPs, which is the first example of using the FRET technique for a bioassay based on UCNPs [52]. In this case, the emission band of the biotinylated $\mathrm{NaYF}_{4}: \mathrm{Yb}$, Er UCNPs overlaps well with the absorption band of the biotinylated AuNPs, thereby quenching occurs in close proximity of both nanoparticles. Consequently, quantitative analysis of avidin could be achieved since the luminescence excited by NIR light was gradually quenched with increasing amounts of avidin. Another biotin-avidin affinity system based on the interaction of two types of nanoparticles was reported by Saleh et al. [53]. By labeling the UCNPs with avidin and the AuNPs with biotin (see Figure 3(b)), they established a model system for a self referenced affinity system by ratioing two emission bands from UCNPs.

The fluorescence-based imaging modality with the aid of FRET is an efficient tool for tracking dynamics of therapeutic drug release. Jiang and Zhang developed the FRETbased UCNPs/siRNA-BOBO3 complex system, which energy is transferred from the donor (UCNPs) to the acceptor (BOBO-3) upon NIR excitation [54]. BOBO-3 is a siRNA staining dye performed by imaging of siRNA. The intracellular changes of siRNA and UCNPs in live cell were observed by FRET between the UCNPs and BOBO-3 in close proximity.

2.2.3. Drug Release. The photoinduced release systems have been suffered from some drawbacks, like they require highenergy UV or visible light as the trigger. However, UV excitation is not optimal for applications in living cells or organisms due to its short penetration depth in biological systems. UCNPs capable of excitation with NIR light and conversion to emit UV light can provide the need of UV light activation in the photolysis and compromise the shortage of UV excitation. Carling et al. developed the remote-control release system by using the decoration of UCNPs with $3^{\prime}, 5^{\prime}$-di(carboxymethoxy)benzoin cage [55]. The rationale behind the choice of $\mathrm{NaYF}_{4}: \mathrm{Yb}, \mathrm{Tm}$ nanoparticles and the photocage is based on the absorption band of $3^{\prime}, 5^{\prime}$ di(carboxymethoxy)benzoin acetate $(\lambda=282 \mathrm{~nm})$ partially overlapping the emission band of the UCNPs $(\lambda=290 \mathrm{~nm})$. The $\mathrm{NaYF}_{4}: \mathrm{Yb}, \mathrm{Tm}$ nanoparticles provided UV light when irradiated with $980 \mathrm{~nm}$ laser followed byenergy transfer to trigger the uncaging in a remote-control process.

2.2.4. Therapy. Most available photosensitizers have absorption bands at wavelengths shorter than $700 \mathrm{~nm}$. Such a photosensitization approach is suitable to treat only superficial tumors due to limited penetration of visible light. For this reason, light delivery is still a challenging issue for deep cancer treatment by PDT. UCNPs can be opted for photosensiters of PDT targeted in tumor at depth in tissue (see Figure 3(c)), owing to the weak tissue absorption of NIR photons which thus can penetrate a few centimeters of tissue. Zhang et al. doped photosensitizer, Merocyanine 540 (MC540) into the thin silica surface of $\mathrm{NaY}_{4}: \mathrm{Yb}, \mathrm{Er}$ and conjugated with a targeted mouse monoclonal antibody (anti-MUC1/episialin), which was specific to MCF-7 breast cancer cells [56]. The singlet oxygen generated from the UCNP of $\mathrm{NaY}_{4}: \mathrm{Yb}, \mathrm{Er} / \mathrm{MC} 540$ upon irradiation with NIR light of $974 \mathrm{~nm}$ was profiled using the compound of 9,10anthracenedipropionic acid (ADPA). This is the first report exploiting UCNP combined with photosensitizer for PDT with NIR excitation. With a further design, Chatterjee et al. synthesized a polyethylenimine- (PEI-) coated UCNP on which the photosensitizer zinc phthalocyanine $(\mathrm{ZnPc})$ could be electrostatically attached [57]. $\mathrm{NaY}_{4}: \mathrm{Yb}, \mathrm{Er}$ was also used to transfer the emitted visible light $(\lambda=670 \mathrm{~nm})$ to excite the attached $\mathrm{ZnPc}$ photosensitizers to generate singlet oxygen upon NIR $980 \mathrm{~nm}$ excitation. Their results show that the treatment of $\mathrm{NaY}_{4}: \mathrm{Yb}, \mathrm{Er} / \mathrm{PEI} / \mathrm{ZnPc}$ can induce effective phototoxicity of HT29 human colonic cells. In order to enhance solubility and increase the efficiency of energy transfer between UCNPs and photosensitizer, the same group synthesized the $\mathrm{NaYF}_{4}$ composite with a mesoporous silica shell encapsulating $\mathrm{ZnPc}$ [58]. In the experiments with MB49 bladder cancer cells pretreated with $\mathrm{NaYF}_{4}: \mathrm{Yb}$, $\mathrm{Er} / \mathrm{ZnPc}$, 5-minute exposure of NIR light was sufficient to activate the energy transfer for generation of abundant singlet oxygen followed by significant cytotoxicity.

Chen and Zhang proposed a novel approach that radiotherapy and PDT are combined and activated synergistically by employing scintillating nanoparticles, and thus the tumor destruction will be more efficient [59]. In this new concept, the photosensitizers such as porphyrins conjugated to $\mathrm{X}$ ray luminescent nanoscintillators are targeted to the tumor. The nanoscintillators convert high-energy $\mathrm{X}$-rays into visible light, which then activates the photosensitizers via energy transfer for PDT. The pilot studies have demonstrated that $\mathrm{LaF}_{3}: \mathrm{Tb}^{3+}$ is an efficient nanoscintillator to activate photosensitizers generating singlet oxygen for PDT [60] (see Figure 3(c)). The energy transfer from $\mathrm{LaF}_{3}: \mathrm{Tb}^{3+}$ nanoparticles to meso-tetra(4-carboxyphenyl) porphine (MTCP) was estimated by the fluorescence quenching technique measured over the lifetime changes. With such an energy transfer mechanism, singlet oxygen was generated from $\mathrm{LaF}_{3}: \mathrm{Tb}^{3+}{ }_{-}$ MTCP conjugates upon X-ray irradiation at a low-energy dose of $13.2 \mathrm{~Gy}$. In addition, the targeting ligands of folic acid conjugated onto the $\mathrm{LaF}_{3}: \mathrm{Tb}^{3+} / \mathrm{MTCP}$ have shown no effect on the singlet oxygen generation.

In the hypoxic environments associated with many tumors, the utility of reactive oxygen species- (ROS-) dependent therapy is limited. To circumvent this problem, Scaffidi et al. have recently developed the anticancer drugs in which scintillating nanoparticles (nanoscintillators) can be used to activate psoralen in deep tissue [61]. Psoralen was bound to a fragment of the HIV-1 TAT cellpenetrating/nuclear targeting peptide anchored to UVAemitting $\mathrm{Y}_{2} \mathrm{O}_{3}$ nanoscintillators. When $\mathrm{X}$-ray radiation is absorbed by the $\mathrm{Y}_{2} \mathrm{O}_{3}$, the emitted UVA light activates psoralen to cross-link adenine and thymine residues in DNA. Although preliminary attempts in the PC-3 human prostate cancer cell line yielded only modest in vitro reductions in cell 
number, their results represent the first evidence of drugassociated, X-ray-activated cell killing or growth inhibition for psoralen-functionalized nanoscintillators.

2.2.5. Others. Rare-earth cerium oxide $\left(\mathrm{CeO}_{2}\right)$ nanoparticles (nanoceria) have shown great potential as antioxidant and radioprotective agents for applications in cancer therapy [62-66]. The autoregenerative reaction involving redox cycles $\mathrm{Ce}^{3+} \leftrightarrow \mathrm{Ce}^{4+}$ oxidation states allows nanoceria to react catalytically with superoxide and hydrogen peroxide, mimicking the behavior of superoxide dismutase and catalase. For this reason, nanoceria can potentially reduce the amount of intracellular ROS and scavenge free radicals produced by radiation, preventing human health from radiation-induced damage. Animal studies by Colon et al. demonstrated that $\mathrm{CeO}_{2}$ nanoparticles protect against radiation-induced pneuminits in animals exposed to high doses of radiation and are well tolerated by living animals [62].

Seal's Group recently synthesized rare earth codoped cerium oxide nanoparticles with a sensitizer $\mathrm{Yb}^{3+}$ and an emitter $\mathrm{Er}^{3+}$ having NIR to visible upconversion fluorescence [67]. The codoped nanoceria exhibited strong upcoverting luminescence with lifetime of $10.6 \mu$ s compared to about $3 \mathrm{~ms}$ of typical UCNPs with fluoride hosts. Although the significant fluorescence quenching occurred on the codoped nanoceria, the intensity is sufficient to use as potential biomarkers. The codoped nanoceria were found to kill lung cancer cells (CRL-5803) as evidenced by determining cell viability and measuring the activation of caspase-3/7. Redoxactive codoped nanoceria may induce apoptosis in cancerous cells by producing ROS and oxidative stress, however, the mechanism is still under investigation. Nevertheless, this work demonstrated an example of the potential using codoped nanoceria for biomedical imaging and therapeutics.

\section{Conclusion}

Different sensing schemes have been developed in which QDs were typically used as FRET donors. To improve the FRET efficiency, how to avoid the aggregation of QDs in vivo is an important issue for their practical use in biology and medicine. In addition, the biocompatibility of QDs remains as a major challenge in advance of their biomedical applications. The development of new class of QDs with low toxicity will be crucial for more versatile applications in biological systems other than optical beacons for in vitro diagnostics (IVDs) and biosensing. The possibility to use rare-earth-doped nanoparticles has been demonstrated in many different biological applications due to their unique optical property and relatively low toxicity. UCNPs can be easily applied in a variety of assay formats including bioimaging, sensing, drug delivery, and cancer therapy. UCNPs offer an alternative with minimal photodamage and autofluorescence due to NIR excitation, which enhances their prospects as optical biolabels. Literatures support the possibility of using scintillating nanoparticles combined with X-ray for self-lighting photodynamic therapy [59]. However, this reported work only demonstrated the capability of scintillating nanoparticles in generation of singlet oxygen in solutions, no cells study was implemented. It is, therefore, particularly interested in further demonstration of the utility of X-ray/PDT combined therapy with in vitro cancer cell lines or even in vivo animal pathological models in the near future. Some efforts have focused on exploring new types of UCNPs conjugated with PS for deep cancer treatment. NIR excitation of such UCNPs to activate photosensitizers also needs further investigation to profile their photodynamic effects in use of cancer therapy in vivo.

\section{Acknowledgment}

This study was prepared with the support of the Grants MED-099-PP-04 and MED-100-PP-04 from the National Health Research Institutes of Taiwan and NSC 99-2113-M400-001-MY3 from the National Science Council of Taiwan.

\section{References}

[1] C. Bouzigues and T. G. Alexandrou, "Biomedical applications of rare-earth based nanoparticles," ACS Nano, vol. 5, no. 11, pp. 8488-8505, 2011.

[2] H. S. Mader, P. Kele, S. M. Saleh, and O. S. Wolfbeis, "Upconverting luminescent nanoparticles for use in bioconjugation and bioimaging," Current Opinion in Chemical Biology, vol. 14, no. 5, pp. 582-596, 2010.

[3] D. K. Chatterjee, M. K. Gnanasammandhan, and Y. Zhang, "Small upconverting fluorescent nanoparticles for biomedical applications," Small, vol. 6, no. 24, pp. 2781-2795, 2010.

[4] F. Wang, D. Banerjee, Y. Liu, X. Chen, and X. Liu, "Upconversion nanoparticles in biological labeling, imaging, and therapy," Analyst, vol. 135, no. 8, pp. 1839-1854, 2010.

[5] M. Haase and H. Schäfer, "Upconverting nanoparticles," Angewandte Chemie International Edition, vol. 50, no. 26, pp. 58085829, 2011.

[6] J. Zhou, Z. Liu, and F. Y. Li, "Upconversion nanophosphors for small-animal imaging," Chemical Society Reviews, vol. 41, no. 3, pp. 1323-1349, 2012.

[7] P. Reiss, M. Protière, and L. Li, "Core/shell semiconductor nanocrystals," Small, vol. 5, no. 2, pp. 154-168, 2009.

[8] U. Resch-Genger, M. Grabolle, S. Cavaliere-Jaricot, R. Nitschke, and T. Nann, "Quantum dots versus organic dyes as fluorescent labels," Nature Methods, vol. 5, no. 9, pp. 763-775, 2008.

[9] I. L. Medintz, H. T. Uyeda, E. R. Goldman, and H. Mattoussi, "Quantum dot bioconjugates for imaging, labelling and sensing," Nature Materials, vol. 4, no. 6, pp. 435-446, 2005.

[10] C. Y. Zhang, H. C. Yeh, M. T. Kuroki, and T. H. Wang, "Singlequantum-dot-based DNA nanosensor," Nature Materials, vol. 4, no. 11, pp. 826-831, 2005.

[11] R. Gill, M. Zayats, and I. Willner, "Semiconductor quantum dots for bioanalysis," Angewandte Chemie International Edition, vol. 47, no. 40, pp. 7602-7625, 2008.

[12] P. Zrazhevskiy, M. Sena, and X. H. Gao, "Designing multifunctional quantum dots for bioimaging, detection, and drug delivery," Chemical Society Reviews, vol. 39, no. 11, pp. 43264354, 2010.

[13] N. Hildebrandt, "Biofunctional quantum dots: controlled conjugation for multiplexed biosensors," ACS Nano, vol. 5, no. 7, pp. 5286-5290, 2011. 
[14] Y. P. Ho, H. H. Chen, K. W. Leong, and T. H. Wang, "Evaluating the intracellular stability and unpacking of DNA nanocomplexes by quantum dots-FRET," Journal of Controlled Release, vol. 116, no. 1, pp. 83-89, 2006.

[15] Y. Choi, J. Lee, K. Kim, H. Kim, P. Sommer, and R. Song, "Fluorogenic assay and live cell imaging of HIV-1 protease activity using acid-stable quantum dot-peptide complex," Chemical Communications, vol. 46, no. 48, pp. 9146-9148, 2010.

[16] J. Lee, Y. Choi, J. Kim, E. Park, and R. Song, "Positively charged compact quantum Dot-DNA complexes for detection of nucleic acids," ChemPhysChem, vol. 10, no. 5, pp. 806-811, 2009.

[17] S. M. Shaheen, H. Akita, A. Yamashita et al., "Quantitative analysis of condensation/decondensation status of pDNA in the nuclear sub-domains by QD-FRET," Nucleic Acids Research, vol. 39, no. 7, article e48, 2011.

[18] C. W. Chi, Y. H. Lao, Y. S. Li, and L. C. Chen, "A quantum dot-aptamer beacon using a DNA intercalating dye as the FRET reporter: application to label-free thrombin detection," Biosensors and Bioelectronics, vol. 26, no. 7, pp. 3346-3352, 2011.

[19] G. Jiang, A. S. Susha, A. A. Lutich, F. D. Stefani, J. Feldmann, and A. L. Rogach, "Cascaded FRET in conjugated polymer/quantum dot/dye-labeled DNA complexes for DNA hybridization detection," ACS Nano, vol. 3, no. 12, pp. 41274131, 2009.

[20] H. H. Chen, Y. P. Ho, X. Jiang, H. Q. Mao, T. H. Wang, and K. W. Leong, "Quantitative comparison of intracellular unpacking kinetics of polyplexes by a model constructed from quantum Dot-FRET," Molecular Therapy, vol. 16, no. 2, pp. 324332, 2008.

[21] H. H. Chen, Y. P. Ho, X. Jiang, H. Q. Mao, T. H. Wang, and K. W. Leong, "Simultaneous non-invasive analysis of DNA condensation and stability by two-step QD-FRET," Nano Today, vol. 4, no. 2, pp. 125-134, 2009.

[22] I. L. Medintz, J. H. Konnert, A. R. Clapp et al., "A fluorescence resonance energy transfer-derived structure of a quantum dot-protein bioconjugate nanoassembly," Proceedings of the National Academy of Sciences of the United States of America, vol. 101, no. 26, pp. 9612-9617, 2004.

[23] J. B. Delehanty, K. Boeneman, C. E. Bradburne, K. Robertson, and I. L. Medintz, "Quantum dots: a powerful tool for understanding the intricacies of nanoparticle-mediated drug delivery," Expert Opinion on Drug Delivery, vol. 6, no. 10, pp. 1091-1112, 2009.

[24] K. Boeneman, B. C. Mei, A. M. Dennis et al., "Sensing caspase 3 activity with quantum dot-fluorescent protein assemblies," Journal of the American Chemical Society, vol. 131, no. 11, pp. 3828-3829, 2009.

[25] K. Boeneman, J. B. Delehanty, K. Susumu, M. H. Stewart, and I. L. Medintz, "Intracellular bioconjugation of targeted proteins with semiconductor quantum dots," Journal of the American Chemical Society, vol. 132, no. 17, pp. 5975-5977, 2010.

[26] K. Boeneman, D. E. Prasuhn, J. B. Blanco-Canosa et al., "Selfassembled quantum dot-sensitized multivalent DNA photonic wires," Journal of the American Chemical Society, vol. 132, no. 51, pp. 18177-18190, 2010.

[27] K. E. Sapsford, J. Granek, J. R. Deschamps et al., "Monitoring botulinum neurotoxin a activity with peptide-functionalized quantum dot resonance energy transfer sensors," ACS Nano, vol. 5, no. 4, pp. 2687-2699, 2011.

[28] H. Yao, Y. Zhang, F. Xiao, Z. Xia, and J. Rao, "Quantum dot/bioluminescence resonance energy transfer based highly sensitive detection of proteases," Angewandte Chemie International Edition, vol. 46, no. 23, pp. 4346-4349, 2007.

[29] R. Freeman, R. Gill, I. Shweky, M. Kotler, U. Banin, and I. Willner, "Biosensing and probing of intracellular metabolic pathways by NADH-sensitive quantum dots," Angewandte Chemie International Edition, vol. 48, no. 2, pp. 309-313, 2009.

[30] T. Skajaa, Y. Zhao, D. J. van den Heuvel et al., "Quantum dot and Cy5.5 labeled nanoparticles to investigate lipoprotein biointeractions via förster resonance energy transfer," Nano Letters, vol. 10, no. 12, pp. 5131-5138, 2010.

[31] V. Bagalkot, L. Zhang, E. Levy-Nissenbaum et al., "Quantum dot-aptamer conjugates for synchronous cancer imaging, therapy, and sensing of drug delivery based on bi-fluorescence resonance energy transfer," Nano Letters, vol. 7, no. 10, pp. 3065-3070, 2007.

[32] F. Auzel, "Upconversion and anti-stokes processes with $\mathrm{f}$ and $\mathrm{d}$ ions in solids," Chemical Reviews, vol. 104, no. 1, pp. 139-173, 2004.

[33] D. K. Chatterjee, A. J. Rufaihah, and Y. Zhang, "Upconversion fluorescence imaging of cells and small animals using lanthanide doped nanocrystals," Biomaterials, vol. 29, no. 7, pp. 937-943, 2008.

[34] R. A. Jalil and Y. Zhang, "Biocompatibility of silica coated $\mathrm{NaYF}_{4}$ upconversion fluorescent nanocrystals," Biomaterials, vol. 29, no. 30, pp. 4122-4128, 2008.

[35] M. Nyk, R. Kumar, T. Y. Ohulchanskyy, E. J. Bergey, and P. N. Prasad, "High contrast in vitro and in vivo photoluminescence bioimaging using near infrared to near infrared up-conversion in $\mathrm{Tm}^{3+}$ and $\mathrm{Yb}^{3+}$ doped fluoride nanophosphors," Nano Letters, vol. 8, no. 11, pp. 3834-3838, 2008.

[36] L. Q. Xiong, Z. G. Chen, M. X. Yu, F. Y. Li, C. Liu, and C. H. Huang, "Synthesis, characterization, and in vivo targeted imaging of amine-functionalized rare-earth up-converting nanophosphors," Biomaterials, vol. 30, no. 29, pp. 5592-5600, 2009.

[37] L. Xiong, Z. Chen, Q. Tian, T. Cao, C. Xu, and F. Li, "High contrast upconversion luminescence targeted imaging in vivo using peptide-labeled nanophosphors," Analytical Chemistry, vol. 81, no. 21, pp. 8687-8694, 2009.

[38] X.-F. Yu, M. Li, M. Y. Xie, L. D. Chen, Y. Li, and Q.-Q. Wang, "Dopant-controlled synthesis of water-soluble hexagonal $\mathrm{NaYF}_{4}$ nanorods with efficient upconversion fluorescence for multicolor bioimaging," Nano Research, vol. 3, no. 1, pp. 51-60, 2010.

[39] X.-F. Yu, Z. Sun, M. Li et al., "Neurotoxin-conjugated upconversion nanoprobes for direct visualization of tumors under near-infrared irradiation," Biomaterials, vol. 31, no. 33, pp. 8724-8731, 2010.

[40] F. Chen, S. Zhang, W. Bu et al., "A neck-formation strategy for an antiquenching magnetic/upconversion fluorescent bimodal cancer probe," Chemistry, vol. 16, no. 37, pp. 11254-11260, 2010.

[41] S. Gai, P. Yang, C. Li et al., "Synthesis of magnetic, up-conversion luminescent, and mesoporous core-shell-structured nanocomposites as drug carriers," Advanced Functional Materials, vol. 20, no. 7, pp. 1166-1172, 2010.

[42] G. Zhang, Y. L. Liu, Q. H. Yuan, C. H. Zong, J. H. Liu, and L. $\mathrm{H}$. Lu, "Dual modal in vivo imaging using upconversion luminescence and enhanced computed tomography properties," Nanoscale, vol. 3, no. 10, pp. 4365-4371, 2011.

[43] J. Zhou, Y. Sun, X. X. Du, L. Q. Xiong, H. Hu, and F. Y. Li, "Dual-modality in vivo imaging using rare-earth nanocrystals 
with near-infrared to near-infrared (NIR-to-NIR) upconversion luminescence and magnetic resonance properties," Biomaterials, vol. 31, no. 12, pp. 3287-3295, 2010.

[44] H. Xu, L. Cheng, C. Wang, X. X. Ma, Y. G. Li, and Z. Liu, "Polymer encapsulated upconversion nanoparticle/iron oxide nanocomposites for multimodal imaging and magnetic targeted drug delivery," Biomaterials, vol. 32, no. 35, pp. 93649373, 2011.

[45] L. Cheng, K. Yang, Y. Li et al., "Facile preparation of multifunctional upconversion nanoprobes for multimodal imaging and dual-targeted photothermal therapy," Angewandte Chemie International Edition, vol. 50, no. 32, pp. 7385-7390, 2011.

[46] L. Wang, P. Li, and L. Wang, "Luminescent and hydrophilic $\mathrm{LaF}_{3}$-polymer nanocomposite for DNA detection," Luminescence, vol. 24, no. 1, pp. 39-44, 2009.

[47] K. Kuningas, T. Rantanen, T. Ukonaho, T. Lövgren, and T. Soukka, "Homogeneous assay technology based on upconverting phosphors," Analytical Chemistry, vol. 77, no. 22, pp. 7348-7355, 2005.

[48] K. Kuningas, T. Ukonaho, H. Päkkilä et al., "Upconversion fluorescence resonance energy transfer in a homogeneous immunoassay for estradiol," Analytical Chemistry, vol. 78, no. 13, pp. 4690-4696, 2006.

[49] K. Kuningas, H. Päkkilä, T. Ukonaho, T. Rantanen, T. Lövgren, and T. Soukka, "Upconversion fluorescence enables homogeneous immunoassay in whole blood," Clinical Chemistry, vol. 53, no. 1, pp. 145-146, 2007.

[50] T. Rantanen, M. L. Järvenpää, J. Vuojola, K. Kuningas, and T. Soukka, "Fluorescence-quenching-based enzyme-activity assay by using photon upconversion," Angewandte Chemie International Edition, vol. 47, no. 20, pp. 3811-3813, 2008.

[51] F. Vetrone, R. Naccache, C. G. Morgan, and J. A. Capobianco, "Luminescence resonance energy transfer from an upconverting nanoparticle to a fluorescent phycobiliprotein," Nanoscale, vol. 2, no. 7, pp. 1185-1189, 2010.

[52] L. Wang, R. Yan, Z. Huo et al., "Fluorescence resonant energy transfer biosensor based on upconversion-luminescent nanoparticles," Angewandte Chemie International Edition, vol. 44, no. 37, pp. 6054-6057, 2005.

[53] S. M. Saleh, R. Ali, T. Hirsch, and O. S. Wolfbeis, "Detection of biotin-avidin affinity binding by exploiting a self-referenced system composed of upconverting luminescent nanoparticles and gold nanoparticles," Journal of Nanoparticle Research, vol. 13, no. 10, pp. 4603-4611, 2011.

[54] S. Jiang and Y. Zhang, "Upconversion nanoparticle-based FRET system for study of siRNA in live cells," Langmuir, vol. 26, no. 9, pp. 6689-6694, 2010.

[55] C.-J. Carling, F. Nourmohammadian, J.-C. Boyer, and N. R. Branda, "Remote-control photorelease of caged compounds using near-infrared light and upconverting nanoparticles," Angewandte Chemie International Edition, vol. 49, no. 22, pp. 3782-3785, 2010.

[56] P. Zhang, W. Steelant, M. Kumar, and M. Scholfield, "Versatile photosensitizers for photodynamic therapy at infrared excitation," Journal of the American Chemical Society, vol. 129, no. 15, pp. 4526-4527, 2007.

[57] D. K. Chatterjee and Z. Yong, "Upconverting nanoparticles as nanotransducers for photodynamic therapy in cancer cells," Nanomedicine, vol. 3, no. 1, pp. 73-82, 2008.

[58] H. S. Qian, H. C. Guo, P. C. L. Ho, R. Mahendran, and Y. Zhang, "Mesoporous-silica-coated up-conversion fluorescent nanoparticles for photodynamic therapy," Small, vol. 5, no. 20, pp. 2285-2290, 2009.
[59] W. Chen and J. Zhang, "Using nanoparticles to enable simultaneous radiation and photodynamic therapies for cancer treatment," Journal of Nanoscience and Nanotechnology, vol. 6, no. 4, pp. 1159-1166, 2006.

[60] Y. Liu, W. Chen, S. Wang, and A. G. Joly, "Investigation of water-soluble $\mathrm{x}$-ray luminescence nanoparticles for photodynamic activation," Applied Physics Letters, vol. 92, no. 4, pp. 043901-043903, 2008.

[61] J. P. Scaffidi, M. K. Gregas, B. Lauly, Y. Zhang, and T. Vo-Dinh, "Activity of psoralen-functionalized nanoscintillators against cancer cells upon X-ray excitation,” ACS Nano, vol. 5, no. 6, pp. 4679-4687, 2011.

[62] J. Colon, L. Herrera, J. Smith et al., "Protection from radiationinduced pneumonitis using cerium oxide nanoparticles," $\mathrm{Na}$ nomedicine, vol. 5, no. 2, pp. 225-231, 2009.

[63] R. W. Tarnuzzer, J. Colon, S. Patil, and S. Seal, "Vacancy engineered ceria nanostructures for protection from radiationinduced cellular damage," Nano Letters, vol. 5, no. 12, pp. 2573-2577, 2005.

[64] C. Korsvik, S. Patil, S. Seal, and W. T. Self, "Superoxide dismutase mimetic properties exhibited by vacancy engineered ceria nanoparticles," Chemical Communications, no. 10, pp. 10561058, 2007.

[65] S. Bhattacharyya, R. A. Kudgus, R. Bhattacharya, and P. Mukherjee, "Inorganic nanoparticles in cancer therapy," Pharmaceutical Research, vol. 28, no. 2, pp. 237-259, 2011.

[66] I. Celardo, J. Z. Pedersen, E. Traversa, and L. Ghibelli, "Pharmacological potential of cerium oxide nanoparticles," Nanoscale, vol. 3, no. 4, pp. 1411-1420, 2011.

[67] S. Babu, J.-H. Cho, J. M. Dowding et al., "Multicolored redox active upconverter cerium oxide nanoparticle for bio-imaging and therapeutics," Chemical Communications, vol. 46, no. 37, pp. 6915-6917, 2010. 

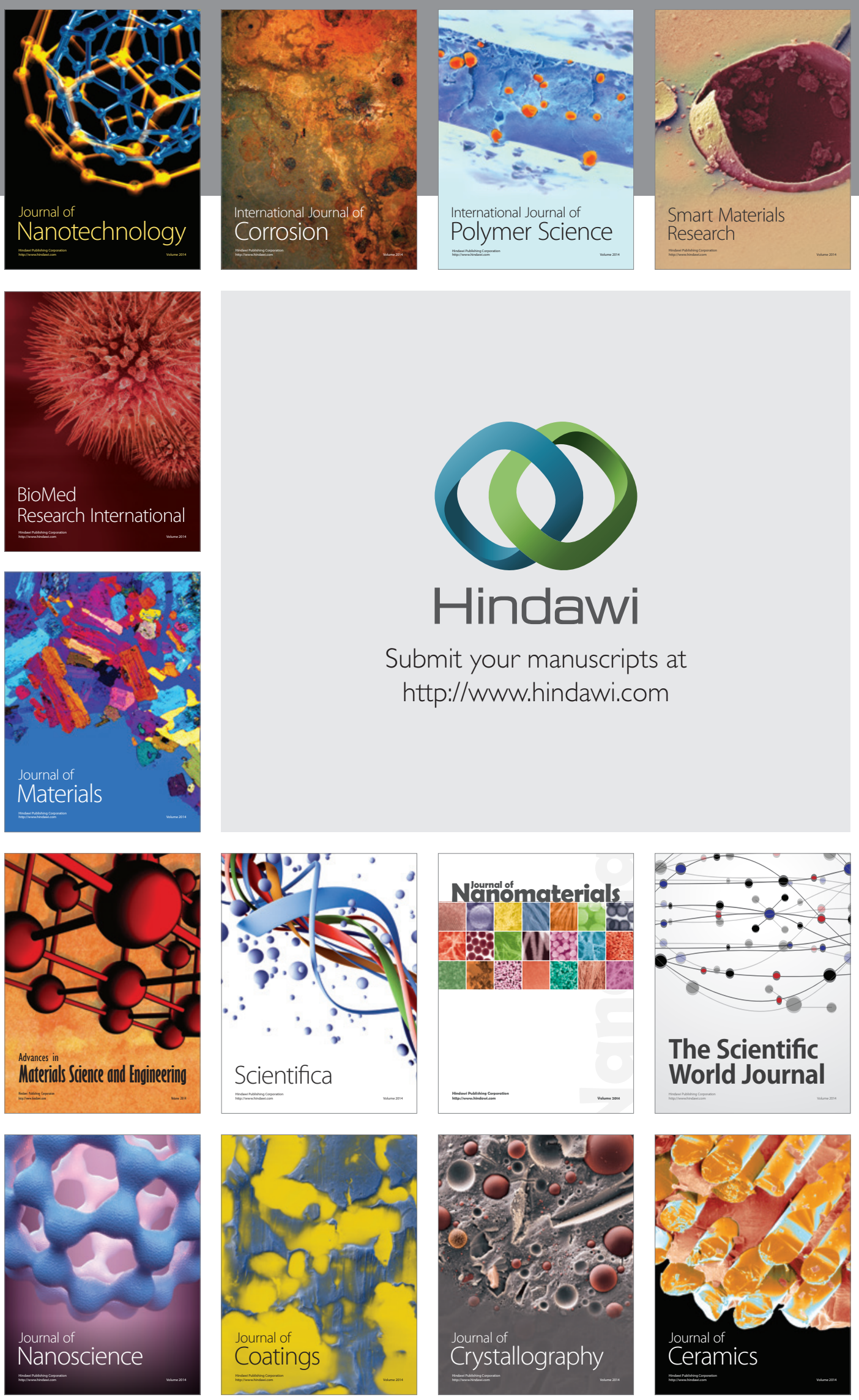

The Scientific World Journal

Submit your manuscripts at

http://www.hindawi.com

\section{World Journal}

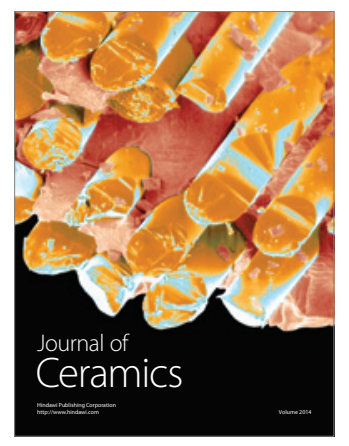

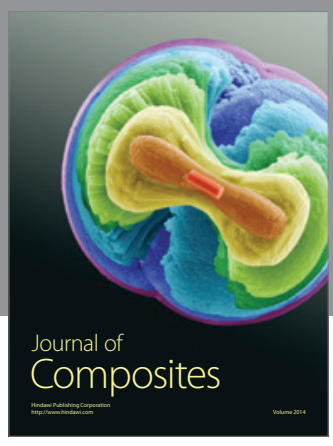
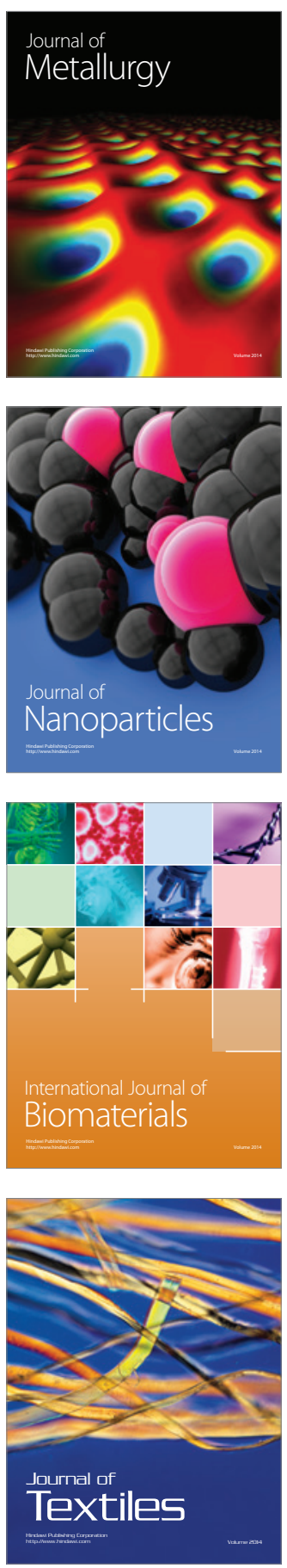